\title{
CONTROLLABILITY TO THE TRAJECTORIES OF PHASE-FIELD MODELS BY ONE CONTROL FORCE*
}

\author{
F. AMMAR KHODJA ${ }^{\dagger}$, A. BENABDALLAH ${ }^{\dagger}$, C. DUPAIX $^{\dagger}$, AND I. KOSTIN $^{\dagger}$
}

\begin{abstract}
In this article, we study the controllability to the trajectories of $2 \times 2$ nonlinear parabolic systems for control forces acting on a single equation of the system. This result, which in particular applies to Caginalp's phase-field model, actually extends those obtained for the semilinear heat equations. The proof relies on Kakutani's fixed point theorem and makes use of an observability estimate for the associated linearized system.
\end{abstract}

Key words. nonlinear parabolic systems, controllability

AMS subject classifications. 35K50, 35K60, 93C20, 93B05

DOI. S0363012902417826

1. Introduction. The aim of this article is the study of controllability for phasefield models when the control force acts on a single equation of the system. The models that we consider here are generalizations of Caginalp's phase-field model [9] (see also [8]) in its enthalpy formulation. For given time $T>0$ and bounded domain $\Omega \subset \mathbb{R}^{N}$ $(1 \leq N<6)$ with smooth boundary $\partial \Omega$, it reads as follows:

$$
\begin{cases}\phi_{t}=\Delta \phi-h(\phi)+u & \\ u_{t}=D \Delta u-\Delta \phi+f & \text { in }(0, T) \times \Omega=Q_{T} \\ \phi=u=0 & \text { on }(0, T) \times \partial \Omega=\Sigma_{T} \\ \phi(0)=\phi_{0}, u(0)=u_{0} & \text { in } \Omega\end{cases}
$$

where $D>0$ is a constant and $h: \mathbb{R} \rightarrow \mathbb{R}$ is a $C^{1}$ function (locally Lipschitz continuous is actually sufficient). We assume that $f=\chi_{\omega} g$, where $g \in L^{2}\left(Q_{T}\right)$ and $\chi_{\omega}$ is the characteristic function of a nonempty open and fixed set $\omega \Subset \Omega$ (namely $\bar{\omega} \subset \Omega$ ). The unknown functions $\phi$ and $u$ can, respectively, be interpreted as a phase parameter and an enthalpy.

As we will see below, a part of the results here obtained depend on the behavior of the function $h$ near $\pm \infty$. However, this hypothesis on $h$ is not a restriction from the phase transition point of view. In fact, besides some monotonicity property of $h$, the main requirement for the phase-field models is that at least for $|s| \leq 1, h(s)=$ $c_{1} H^{\prime}(s)-c_{2} s$, where $c_{1}, c_{2}$ are positive constants and $H$ is a symmetric double-well potential having two local minima at $s= \pm 1$. Although this model is known to be inconsistent with the second law of thermodynamics, it has been proved to be quite useful since many other models of the phase transition phenomena can be derived from Caginalp's model by taking suitable limits with respect to the parameters of the system (i.e., $D, c_{1}$, and $c_{2}$ ).

In this work we are interested in the controllability of such systems and we recall, therefore, that system (1) is said to be controllable to the trajectories at time $T$ if for any initial data $\left(\phi_{0}, u_{0}\right)$ there exists a control $f$ such that the corresponding solution

\footnotetext{
*Received by the editors November 14, 2002; accepted for publication (in revised form) April 2, 2003; published electronically November 6, 2003.

http://www.siam.org/journals/sicon/42-5/41782.html

†Université de Franche-Comté, Département de Mathématiques, CNRS-UMR 6623, 16 Route de Gray, 25030 Besançon Cedex, France (ammar@descartes.univ-fcomte.fr, assia@descartes.univfcomte.fr, dupaix@descartes.univ-fcomte.fr, kostin@descartes.univ-fcomte.fr).
} 
$(\phi, u)$ of $(1)$ is defined on $[0, T]$ and satisfies

$$
(\phi(T, .), u(T, .))=\left(\phi^{*}(T, .), u^{*}(T, .)\right) \text { a.e. in } \Omega,
$$

where $\left(\phi^{*}, u^{*}\right)$ is any bounded solution of $(1)$ defined on $[0, T]$ associated with the data $\phi_{0}^{*}, u_{0}^{*}$, and $f^{*}$.

System (1) is locally controllable to the trajectories at time $T$ if there exists a constant $r>0$ such that for any initial data $\left(\phi_{0}, u_{0}\right)$ satisfying $\left\|\phi_{0}-\phi_{0}^{*}\right\|+\left\|u_{0}-u_{0}^{*}\right\|<$ $r$ the solution $(\phi, u)$ of system (1) satisfies (2).

The functional spaces will be precised in the forthcoming sections.

Considering $\left(\phi-\phi^{*}, u-u^{*}\right)$ as a new unknown, the problem is reduced to drive the solution at time $T$ to the state $(0,0)$. Therefore, referring to the linear case, this kind of controllability is often called null-controllability. Notice that in the finite dimensional case and for linear problems, null, exact, and approximate controllability are equivalent (see, for instance, [21]).

The originality of our approach is to control $2 \times 2$ nonlinear systems by acting (locally in space) on a single equation of it. From this point of view and to our knowledge, our results are the first ones in this direction. Controlling a system with a minimum number of forces, or by forces satisfying an algebraic or a differential (or any other type) relation, is a common problem in the control theory (see, for instance, [19, Chapter V, p. 322] for other types of systems). Other approaches deal with dynamic controls which, roughly speaking, means that the control itself obeys a dynamic (see [1] and the references therein). From this last point of view, the variable $u$ of system (1) can be seen as a dynamic control with respect to the variable $\phi$.

Null-controllability of linear and semilinear heat equations has been extensively studied in recent years. The main ingredients, therefore, are global estimates of Carleman type for linear parabolic equations with an additional fixed point argument for the nonlinear case. The appropriate version of the Carleman estimates was established, in their most general form, by Imanuvilov [17] and Fursikov and Imanuvilov [16] (see also Lebeau and Robbiano [20] for the linear heat equation). These estimates were first used to prove the controllability to the trajectories of semilinear heat equations with globally Lipschitz continuous nonlinearities (see [16]) and then to extend this property to superlinear heat equations (see, for instance, [12], [5], [14]). In contrast with this collection of results, few works deal with the controllability of linear or nonlinear parabolic systems. In [10] De Teresa considers a semilinear heat equation coupled with an adjoint problem. The specific form of the system allows the proof of controllability to the trajectories in the case of globally Lipschitz continuous nonlinearities. However, in the case of system (1) such a proof does not work since the two equations are coupled in a very different way. Recently, Anita and Barbu [3] have considered a $2 \times 2$ reaction-diffusion system with a bilinear nonlinearity. They proved the local controllability to the stationary solutions of the system by two control forces acting on both equations of the system. Their result seems to be the first one dealing with the controllability to the trajectories for semilinear parabolic systems. More recently, Barbu [6] has considered the phase-field model (1) with a cubic nonlinearity and in an (equivalent) temperature formulation. He has proved the local controllability to the stationary solutions by two control forces localized on the same subdomain. At this stage let us point out that the one control force result cannot be obtained as a simple generalization of Barbu's result. It relies on the construction (using a multiplier technique) of a suitable functional $\Lambda$ with suitable weights (see Lemma 3.4 and (34)). This is one of the crucial points of the proof of our results. It 
also appears to be useful for the study of the null-controllability by a single force of abstract general linear "parabolic" systems of two equations [2]. Besides the sufficient conditions of controllability, in [2] we also construct a counterexample showing that if the coupling operators are "too compact," null-controllability fails to hold.

The sketch of the proof of our results is by now well known and was adopted for a scalar semilinear parabolic equation by several authors (see [16], [12], [5], [14]). The idea is to prove an observability estimate for the "linearized" system and then to use a fixed point theorem. The main difficulty is to prove the observability estimate for the "linearized" system corresponding to the control by a single force. To achieve this goal we first establish a global Carleman-type estimate for a linear parabolic system. This implies the controllability by two forces. In a second step we obtain an observability estimate which implies the controllability of the linear system by a single force. The fixed point method we use makes it necessary to construct a sufficiently regular control which induces by parabolic regularity a solution in a suitable space (here $L^{\infty}\left(Q_{T}\right)$ ). This explains the need for a "refined" observability estimate (see Lemma 3.4).

The paper is organized as follows. In the second section, by admitting an essential lemma (see Lemma 2.3), we state and prove the main result of this article (Theorem 2.1). We prove this lemma in the fourth section, after having shown in the third section the crucial observability estimate for a linear adjoint problem (see Lemma 3.4).

We conclude this section with the following two remarks.

1. Since the diffusion coefficient $D$ in (1) changes only the constants in the proofs, we assume for simplicity that $D=1$. However, the dependence of these constants on $D$ (and on $c_{1}$ and $c_{2}$ for Caginalp's model) is explicit and may be of interest from the point of view of the theory of phase transitions.

2. Also for the sake of simplicity, we consider only the homogeneous Dirichlet boundary conditions. However, all the results seem to still be valid for the homogeneous Neumann boundary conditions.

2. The main result. To simplify, we will work with a function $h$ satisfying

$$
h \in C^{1}(R), h(0)=0 .
$$

With this assumption, system (1) admits $(0,0)$ as a global solution associated with null initial data and control.

Let $1 \leq N<6$ and $\frac{N+2}{2}<q_{N}<2 \frac{N+2}{N-2}$ if $N \geq 3$, and $q_{N} \in(2,+\infty)$ if $N=1,2$ (even if the physical case reduces to $N=1,2,3$ ). We use the following notation:

$$
W_{q}^{2,1}\left(Q_{T}\right)=\left\{\zeta \in L^{q}\left(Q_{T}\right) ; D_{t}^{r} D_{x}^{s} \zeta \in L^{q}\left(Q_{T}\right) ; 2 r+s \leq 2\right\}
$$

and $W^{m, p}(\Omega)$ is the standard Sobolev space. With the assumption on $q_{N}$, we have the following embeddings [18]:

$$
W_{q_{N}}^{2,1}\left(Q_{T}\right) \hookrightarrow L^{\infty}\left(Q_{T}\right), \quad W^{2\left(1-\frac{1}{q_{N}}\right), q_{N}}(\Omega) \hookrightarrow L^{\infty}(\Omega) .
$$

Our main result is the following theorem.

Theorem 2.1. Assume that $h$ satisfies (3). Let $T>0,1 \leq N<6$, and let $\left(\phi^{*}, u^{*}\right)$ be a globally defined and bounded solution of (1) associated with the data $\phi_{0}^{*}, u_{0}^{*} \in L^{2}(\Omega)$ and $g^{*} \in L^{2}\left(Q_{T}\right)$.

(i) Controllability to the trajectories. If $h$ satisfies

$$
\lim _{|s| \rightarrow+\infty} \frac{h(s)}{|s| \ln ^{3 / 2}(1+|s|)}=0,
$$


then $\forall \phi_{0}, u_{0} \in L^{2}(\Omega)$ with $\phi_{0}-\phi^{*}, u_{0}-u_{0}^{*} \in H_{0}^{1}(\Omega) \cap W^{2\left(1-\frac{1}{q_{N}}\right), q_{N}}(\Omega)$, one can find $g \in L^{2}\left(Q_{T}\right)$ with $g-g^{*} \in L^{q_{N}}\left(Q_{T}\right)$ such that there exists $\left(\phi_{g}, u_{g}\right)$ solution of (1) with $\phi_{g}-\phi^{*}, u_{g}-u^{*} \in W_{q_{N}}^{2,1}\left(Q_{T}\right)$ and satisfying

$$
\left(\phi_{g}-\phi^{*}\right)(T)=0, \quad\left(u_{g}-u^{*}\right)(T)=0 .
$$

(ii) Local controllability to the trajectories. There is $\rho>0$ such that if $\phi_{0}-$ $\phi^{*}, u_{0}-u_{0}^{*} \in H_{0}^{1}(\Omega) \cap W^{2\left(1-\frac{1}{q_{N}}\right), q_{N}}(\Omega)$ with $\left\|\left(\phi_{0}-\phi^{*}, u_{0}-u_{0}^{*}\right)\right\|_{L^{\infty}(\Omega)} \leq \rho$, one can find $g-g^{*} \in L^{q_{N}}\left(Q_{T}\right)$ such that there exists $\left(\phi_{g}, u_{g}\right)$ solution of (1) with $\phi_{g}-\phi^{*}, u_{g}-u^{*} \in W_{q_{N}}^{2,1}\left(Q_{T}\right)$ and satisfying

$$
\left(\phi_{g}-\phi^{*}\right)(T)=0, \quad\left(u_{g}-u^{*}\right)(T)=0 .
$$

Remark 2.2. This result implies the one of Barbu [6, Theorem 1, p. 364].

Proof of Theorem 2.1. For the sake of simplicity, we assume that $\phi_{0}^{*}=u_{0}^{*}=0$ and $g^{*}=0$ so that $\left(\phi^{*}, u^{*}\right)=(0,0)$, and we introduce the function:

$$
\nu(s)=\left\{\begin{array}{cc}
\frac{h(s)}{s} & \text { if } s \neq 0 \\
h^{\prime}(0) & \text { if } s=0 .
\end{array}\right.
$$

For $R>0$, we set

$$
K_{R}=\left\{z \in L^{\infty}\left(Q_{T}\right) ;\|z\|_{L^{\infty}\left(Q_{T}\right)} \leq R\right\}
$$

and let $z \in K_{R}$.

Consider the "linearized" version of (1) with $a=-\nu(z)$ :

$$
\begin{cases}\psi_{t}=\Delta \psi+a \psi+v & \text { in } Q_{T}, \\ v_{t}=\Delta v-\Delta \psi+\chi_{\omega} g & \text { in } Q_{T} \\ \psi=v=0 & \text { on } \Sigma_{T} \\ \psi(0, .)=\psi_{0}, v(0, .)=v_{0} & \text { in } \Omega .\end{cases}
$$

Note that if $\left(\phi^{*}, u^{*}\right) \neq(0,0)$, it suffices to consider the function

$$
\begin{aligned}
\widetilde{\nu} & : Q_{T} \times \mathbb{R} \rightarrow \mathbb{R}, \\
\widetilde{\nu}(t, x, s) & =\left\{\begin{array}{cc}
\frac{h\left(s+\phi^{*}\right)-h\left(\phi^{*}\right)}{h^{\prime}\left(\phi^{*}\right)} & \text { if } s \neq 0, \\
\text { if } s=0
\end{array}\right.
\end{aligned}
$$

and to study (6) with $a(t, x)=-\widetilde{\nu}(t, x, z)$. The crucial point is the following result.

Lemma 2.3. Let $X_{0}=\left(\psi_{0}, v_{0}\right) \in\left(H_{0}^{1}(\Omega) \cap W^{2\left(1-\frac{1}{q_{N}}\right), q_{N}}(\Omega)\right)^{2}$. For any $T>$ 0 , there exists $g \in L^{q_{N}}\left(Q_{T}\right)$ such that the associated solution $\psi_{g}, v_{g}$ of (6) is in $L^{2}\left(0, T ; H_{0}^{1}(\Omega)\right) \cap W_{q_{N}}^{2,1}\left(Q_{T}\right)$ and, moreover,

$$
\begin{aligned}
\left(\psi_{g}, v_{g}\right)(T) & =0 \quad \text { a.e. } \Omega \\
\left\|\chi_{\omega} g\right\|_{L^{q_{N}}\left(Q_{T}\right)}^{2} & \leq C_{T} \quad\left\|X_{0}\right\|_{L^{2}(\Omega)}^{2},
\end{aligned}
$$

with

$$
C_{T}=\exp \left(C\left(1+\frac{1}{T}+\left(1+\|a\|_{\infty}\right) T+\|a\|_{\infty}^{2 / 3}\right)\right) .
$$

The next sections are devoted to the proof of this lemma. With this result in hand, let us continue the proof of Theorem 2.1. 
For each $z \in K_{R}$, we apply Lemma 2.3 and consider the set $\digamma(z) \subset L^{2}\left(Q_{T}\right)$ of the first components $\psi_{g}$ of all the solutions $\psi_{g}, v_{g} \in L^{2}\left(0, T ; H_{0}^{1}(\Omega)\right) \cap W_{q_{N}}^{2,1}\left(Q_{T}\right)$ associated with any control $g \in L^{q_{N}}\left(Q_{T}\right)$ such that $\left(\psi_{g}, v_{g}\right)(T)=0$ a.e. $\mathrm{s} \Omega$ and $\left\|\chi_{\omega} g\right\|_{L^{q_{N}\left(Q_{T}\right)}}^{2} \leq C_{T}\left\|X_{0}\right\|_{L^{2}(\Omega)}^{2}$. The set is a nonempty closed convex subset of $L^{2}\left(Q_{T}\right)$.

Let us prove that for $R>0$ sufficiently large we have $\digamma\left(K_{R}\right) \subset K_{R}$. To do this, we first show that

$$
\left\|\psi_{g}\right\|_{L^{\infty}\left(Q_{T}\right)}^{2} \leq\left\|\left(\psi_{g}, v_{g}\right)\right\|_{L^{\infty}\left(Q_{T}\right)}^{2} \leq C_{T}\left\|X_{0}\right\|_{L^{\infty}(\Omega)}^{2} .
$$

Indeed, using the fundamental matrix $\Gamma=\left(\Gamma_{i j}\right)_{1 \leq i, j \leq 2}$ associated with the parabolic operator

$$
L_{0}=\left(\begin{array}{cc}
\partial_{t}-\Delta & 0 \\
\Delta & \partial_{t}-\Delta
\end{array}\right)
$$

(see [18, p. 620]), with homogeneous Dirichlet boundary conditions, we can write down the solution of $(6)$ with the notation $X_{g}=\left(\psi_{g}, v_{g}\right)$

$$
\begin{aligned}
X_{g}(t, x)= & \int_{\Omega} \Gamma(t, 0, x, y) X_{0}(y) d y \\
& +\int_{0}^{t} \int_{\Omega} \Gamma(t, \tau, x, y)\left(a \psi_{g}+v_{g}, \chi_{\omega} g\right)(\tau, y) d y d \tau .
\end{aligned}
$$

The entries $\Gamma_{i j}$ of $\Gamma$ satisfy for $1 \leq i, j \leq 2$

$$
\begin{array}{r}
\left|D_{t}^{k} D_{x}^{s} \Gamma_{i j}\right| \leq C(t-\tau)^{-\frac{N+2 k+s}{2}} \exp \left(-C \frac{|x-y|^{2}}{t-\tau}\right), \\
x, y \in \Omega, 0<\tau<t<T, \quad 2 s+k=0,1,2
\end{array}
$$

(notice that in this special situation, $\Gamma_{12}=0$ ). The positive constant $C$ depends only on $\Omega$ and $1 \leq i, j \leq 2$. We will assume, without loss of generality, that it is the same constant. Set

$$
\Gamma_{0}(t, x)=t^{-\frac{N}{2}} \exp \left(-C \frac{|x|^{2}}{t}\right), \quad(t, x) \in(0, \infty) \times \mathbb{R}^{N} .
$$

We know by [18, Theorem 10.4] that $X_{g} \in W_{q_{N}}^{2,1}\left(Q_{T}\right)$ and the embeddings (4) allow us to write

$$
\begin{aligned}
\left\|X_{g}(t)\right\|_{L^{\infty}(\Omega)} \leq & C\left(\left\|\Gamma_{0}(t, .) *\left|X_{0}\right|\right\|_{L^{\infty}(\Omega)}+\left\|\Gamma_{0} \underset{t, x}{*} \chi_{\omega}|g|\right\|_{L^{\infty}(\Omega)}\right. \\
& \left.+\left(1+\|a\|_{\infty}\right)\left\|\Gamma_{0} \underset{t, x}{*}\left|X_{g}\right|\right\|_{L^{\infty}(\Omega)}\right),
\end{aligned}
$$

where $(f \underset{t, x}{*} g)(t, x)=\int_{0}^{t} d \tau \int_{\Omega} f(t-\tau, x-y) d y$. Now, we first have

$$
\begin{aligned}
\left\|\Gamma_{0}(t, .) *\left|X_{0}\right|\right\|_{L^{\infty}(\Omega)} & \leq\left\|\Gamma_{0}(t, .)\right\|_{L^{1}(\Omega)}\left\|X_{0}\right\|_{L^{\infty}(\Omega)} \\
& \leq C\left\|X_{0}\right\|_{L^{\infty}(\Omega)}
\end{aligned}
$$


since for any $t>0,\left\|\Gamma_{0}(t, .)\right\|_{L^{1}(\Omega)} \leq C$ (a constant depending only on the dimension $N)$ and, in the same way,

$$
\left\|\Gamma_{0} \underset{t, x}{*}\left|X_{g}\right|\right\|_{L^{\infty}(\Omega)} \leq C \int_{0}^{t}\left\|X_{g}(\tau)\right\|_{L^{\infty}(\Omega)} d \tau .
$$

From Young's inequality (see [7]), we get with $\frac{1}{p}+\frac{1}{q_{N}}=1$ and the condition on $q_{N}$ :

$$
\begin{aligned}
\left\|\Gamma_{0} \underset{t, x}{*} \chi_{\omega}|g|\right\|_{L^{\infty}(\Omega)} & \leq\left\|\Gamma_{0} \underset{t, x}{*} \chi_{\omega}|g|\right\|_{L^{\infty}\left(Q_{t}\right)} \\
& \leq\left\|\Gamma_{0}\right\|_{L^{p}\left(Q_{t}\right)}\left\|\chi_{\omega} g\right\|_{L^{q_{N}}\left(Q_{t}\right)} \\
& \leq C T^{-\frac{N+2}{2 q_{N}}+1}\left\|\chi_{\omega} g\right\|_{L^{q_{N}\left(Q_{T}\right)}} .
\end{aligned}
$$

These three last inequalities transform (11) into

$$
\begin{aligned}
\left\|X_{g}(t)\right\|_{L^{\infty}(\Omega)} \leq & C\left(\left\|X_{0}\right\|_{L^{\infty}(\Omega)}+T^{-\frac{N+2}{2 q_{N}}+1}\left\|\chi_{\omega} g\right\|_{L^{q_{N}}\left(Q_{T}\right)}\right. \\
& \left.+\left(1+\|a\|_{L^{\infty}\left(Q_{T}\right)}\right) \int_{0}^{t}\left\|X_{g}(\tau)\right\|_{L^{\infty}(\Omega)} d \tau\right),
\end{aligned}
$$

and from Gronwall's inequality we get

$$
\left\|X_{g}\right\|_{L^{\infty}\left(Q_{T}\right)} \leq C e^{C\left(1+\|a\|_{L^{\infty}\left(Q_{T}\right)}\right) T}\left(\left\|X_{0}\right\|_{L^{\infty}(\Omega)}+T^{-\frac{N+2}{2 q_{N}}+1}\left\|\chi_{\omega} g\right\|_{L^{q_{N}}\left(Q_{T}\right)}\right),
$$

and (9) follows from (12) and Lemma 2.3.

Now, from (5) it follows that for any $\eta>0$ there exists $C_{\eta}=C_{\eta}(h)>0$ such that

$$
|\nu(s)|^{2 / 3} \leq C_{\eta}+\eta \ln (1+|s|) \forall s \in \mathbb{R}
$$

and

$$
\begin{aligned}
\left\|\psi_{g}\right\|_{L^{\infty}\left(Q_{T}\right)}^{2} \leq & \exp \left(C\left(1+\frac{1}{T}+\left(1+\|\nu(z)\|_{L^{\infty}\left(Q_{T}\right)}\right) T+\|\nu(z)\|_{L^{\infty}\left(Q_{T}\right)}^{2 / 3}\right)\right)\left\|X_{0}\right\|_{L^{\infty}(\Omega)}^{2} \\
\leq & \exp \left(C \left(1+\frac{1}{T}+\left(1+\left[C_{\eta}+\eta \ln (1+R)\right]^{3 / 2}\right) T\right.\right. \\
& \left.\left.+C_{\eta}+\eta \ln (1+R)\right)\right)\left\|X_{0}\right\|_{L^{\infty}(\Omega)}^{2} .
\end{aligned}
$$

Choosing $T:=T(R, \eta)=\left[C_{\eta}+\eta \ln (1+R)\right]^{-1}$, we get for $R$ sufficiently large

$$
\begin{aligned}
\left\|\psi_{g}\right\|_{L^{\infty}\left(Q_{T}\right)}^{2} & \leq \exp \left(C\left(1+C_{\eta}+\eta \ln (1+R)\right)\right)\left\|X_{0}\right\|_{L^{\infty}(\Omega)}^{2} \\
& \leq(1+R)^{\eta C} \exp \left(C\left(1+C_{\eta}\right)\right)\left\|X_{0}\right\|_{L^{\infty}(\Omega)}^{2} .
\end{aligned}
$$

Choosing $\eta=\frac{1}{2 C}$ yields

$$
\left\|\psi_{g}\right\|_{L^{\infty}\left(Q_{T}\right)}^{2} \leq C(1+R)^{1 / 2}\left\|X_{0}\right\|_{L^{\infty}(\Omega)}^{2},
$$

and clearly this will imply, for $R$ sufficiently large, that

$$
\left\|\psi_{g}\right\|_{L^{\infty}\left(Q_{T}\right)}^{2} \leq R .
$$


It follows that $\digamma\left(K_{R}\right) \subset K_{R}$. Parabolic regularity implies that $\digamma\left(K_{R}\right)$ is relatively compact in $L^{2}\left(Q_{T}\right)$ and exactly as in [5], and $\digamma$ is semicontinuous using again [18, Theorem 10.4]. Applying the Kakutani fixed point theorem (see, for instance, [4]) in the space $L^{2}\left(Q_{T}\right)$, we deduce that there is at least one $z \in L^{\infty}\left(Q_{T}\right)$ such that $z \in \digamma(z)$. Therefore, there is at least one pair $\left(\psi_{g}, v_{g}\right)$ satisfying the first claim of Theorem 2.1. Actually, we have proved this first claim for any $T=T(R, \eta)$, but, clearly, this implies the same result for any $T>T(R, \eta)$ : in this case, we choose a control defined on $(0, T(R, \eta))$ which gives a solution satisfying $(7)$ at $T=T(R, \eta)$ and extend it by 0 to the whole interval $(0, T)$.

The second claim of Theorem 2.1 is obtained starting from (9) and choosing $X_{0}$ so that $C_{T}\left\|X_{0}\right\|_{L^{\infty}(\Omega)}^{2} \leq R$. This ends the proof of Theorem 2.1.

3. Observability estimate. The main result of this section is the proof of the observability estimate (32) in Lemma 3.4 which allows us to build controls satisfying estimate (8) of Lemma 2.3.

Following [15], let us introduce some notation. Let $\omega^{\prime} \Subset \omega$ be a subdomain of $\omega$ and let $\beta$ be a $C^{2}(\bar{\Omega})$ function such that

$$
\min \left\{|\nabla \beta(x)|, x \in \overline{\Omega \backslash \omega^{\prime}}\right\}>0 \quad \text { and } \quad \frac{\partial \beta}{\partial n} \leq 0 \text { on } \partial \Omega,
$$

where $n$ denotes the outward unit normal to $\partial \Omega$. Moreover, we can always assume that $\beta$ satisfies

$$
\min \{\beta(x), x \in \bar{\Omega}\} \geq \max \left(\frac{3}{4}\|\beta\|_{L^{\infty}(\Omega)}, \ln (3)\right) .
$$

Finally, we introduce the following functions with parameters $\lambda>0$ and $\tau>0$ :

$$
\begin{aligned}
\rho(t, x) & :=\frac{e^{\lambda \beta(x)}}{t(T-t)}, \quad(t, x) \in Q_{T}, \\
\alpha(t, x): & =\tau \frac{e^{\frac{4}{3} \lambda\|\beta\|_{L} \infty(\Omega)}-e^{\lambda \beta(x)}}{t(T-t)}, \quad(t, x) \in Q_{T} .
\end{aligned}
$$

Then the following result holds (Carleman estimate).

Theorem 3.1 (see [15, Theorem 7.1, p. 288]). There exist $\lambda_{0}>0, \tau_{0}>0$ and a positive constant $C$ such that $\forall \lambda \geq \lambda_{0}, \forall \tau \geq \tau_{0}$, and $\forall s \geq-3$ the inequality

$$
\begin{aligned}
& \int_{Q_{T}}\left(\frac{1}{\lambda}\left|z_{t}\right|^{2}+\left|D_{x}^{2} z\right|^{2}+\lambda \tau^{2} \rho^{2}|\nabla z|^{2}+\lambda^{4} \tau^{4} \rho^{4} z^{2}\right) \rho^{2 s-1} e^{-2 \alpha} d x d t \\
\leq & C\left(\tau \int_{Q_{T}}\left|z_{t} \pm \Delta z\right|^{2} \rho^{2 s} e^{-2 \alpha} d x d t+\lambda^{4} \tau^{4} \int_{0}^{T} \int_{\omega^{\prime}} z^{2} \rho^{2 s+3} e^{-2 \alpha} d x d t\right)
\end{aligned}
$$

holds for any function $z$ satisfying an homogeneous Dirichlet condition and such that the right-hand side of (18) is finite. Moreover, the constants $C$ and $\lambda_{0}$ depend only on $\Omega$ and $\omega^{\prime}$. The constant $\tau_{0}$ is of the form

$$
\tau_{0}=c_{0}\left(\Omega, \omega^{\prime}\right)\left(T+T^{2}\right) .
$$

The explicit dependence in time of the constants is not given in [15]. We refer to [13], where the above formula for $\tau_{0}$ is obtained. 

and $a$.

In what follows, the symbol $C$ will stand for various constants independent of $T$

The adjoint problem associated with (6) is

$$
\begin{cases}\varphi_{t}=\Delta \varphi+a \varphi-\Delta w & \text { in }(0, T) \times \Omega=Q_{T} \\ w_{t}=\Delta w+\varphi & \text { in } Q_{T}, \\ \varphi=w=0 & \text { on }(0, T) \times \partial \Omega=\Sigma_{T} \\ \varphi(0)=\varphi_{0}, w(0)=w_{0}, & \text { in } \Omega\end{cases}
$$

Let us introduce the following notation: For given $\lambda$ and $\tau$ as in Theorem 3.1, we set $\delta=\tau \rho$ and consider the functional:

$$
I(s, z)=\int_{Q_{T}}\left(\frac{1}{\lambda}\left|z_{t}\right|^{2}+|\Delta z|^{2}+\lambda \delta^{2}|\nabla z|^{2}+\lambda^{4} \delta^{4} z^{2}\right) \delta^{2 s-1} e^{-2 \alpha} d x d t .
$$

LEMMA 3.2. Let $\lambda_{0}>1, \tau_{1}=C\left(T+\left(1+\|a\|_{L^{\infty}\left(Q_{T}\right)}^{2 / 3}\right) T^{2}\right)$, $C$ being the constant given in Theorem 3.1. Then $\forall \lambda \geq \lambda_{0}, \forall \tau \geq \tau_{1}$, and $\forall s \geq-3 / 2$, the solution $(\varphi, w)$ of (19) satisfies the estimate:

$$
I\left(s-\frac{3}{2}, \varphi\right)+I(s, w) \leq C\left(1+\lambda^{4}\right) \int_{0}^{T} \int_{\omega^{\prime}}\left(\varphi^{2} \delta^{2 s}+w^{2} \delta^{2 s+3}\right) e^{-2 \alpha} d x d t .
$$

As a consequence, we get

$$
I(-3, \varphi)+I\left(-\frac{3}{2}, w\right) \leq C\left(1+\lambda^{4}\right) \int_{0}^{T} \int_{\omega^{\prime}}\left(\varphi^{2}+w^{2}\right) e^{-2 \alpha} d x d t
$$

Remark 3.3. The estimate (22) is sufficient to prove controllability by two forces as in Barbu [6].

Proof. Applying Theorem 3.1 to the first equation of problem (6) and multipyling (18) by $\tau^{2 s-1}$, we obtain

$$
\begin{aligned}
I(s, \varphi) \leq & C\left(\int_{Q_{T}}\left(|\Delta w|^{2}+|a \varphi|^{2}\right) \delta^{2 s} e^{-2 \alpha} d x d t\right. \\
& \left.+\lambda^{4} \int_{0}^{T} \int_{\omega^{\prime}} \varphi^{2} \delta^{2 s+3} e^{-2 \alpha} d x d t\right) .
\end{aligned}
$$

Theorem 3.1 applied to the second equation of (6) yields

$$
\begin{aligned}
\int_{Q_{T}}|\Delta w|^{2} \delta^{2 s} e^{-2 \alpha} d x d t \leq & I\left(s+\frac{1}{2}, w\right) \\
\leq & C\left(\int_{Q_{T}} \varphi^{2} \delta^{2 s+1} e^{-2 \alpha} d x d t\right. \\
& \left.+\lambda^{4} \int_{0}^{T} \int_{\omega^{\prime}} w^{2} \delta^{2 s+4} e^{-2 \alpha} d x d t\right) .
\end{aligned}
$$

Inserting the latter estimate in (23), we get

$$
\begin{aligned}
I(s, \varphi) \leq & C\left(\int_{Q_{T}}\left(\varphi^{2} \delta^{2 s+1}+|a \varphi|^{2} \delta^{2 s}\right) e^{-2 \alpha} d x d t\right. \\
& \left.+\lambda^{4} \int_{0}^{T} \int_{\omega^{\prime}}\left(\varphi^{2} \delta^{2 s+3}+w^{2} \delta^{2 s+4}\right) e^{-2 \alpha} d x d t\right) .
\end{aligned}
$$


Observe that

$$
\begin{aligned}
& \int_{Q_{T}}\left(\delta^{2 s+1} \varphi^{2}+\delta^{2 s}|a \varphi|^{2}\right) e^{-2 \alpha} d x d t \\
\leq & \int_{Q_{T}}\left(\delta^{2 s+1} \varphi^{2}+\|a\|_{L^{\infty}\left(Q_{T}\right)}^{2} \delta^{2 s}|\varphi|^{2}\right) e^{-2 \alpha} d x d t .
\end{aligned}
$$

Thus, we get

$$
\begin{aligned}
I(s, \varphi) \leq & C\left(\int_{Q_{T}}\left(\delta^{2 s+1} \varphi^{2}+\|a\|_{L^{\infty}\left(Q_{T}\right)}^{2} \delta^{2 s}|\varphi|^{2}\right) e^{-2 \alpha} d x d t\right. \\
& \left.+\lambda^{4} \int_{0}^{T} \int_{\omega^{\prime}}\left(\varphi^{2} \delta^{2 s+3}+w^{2} \delta^{2 s+4}\right) e^{-2 \alpha} d x d t\right) .
\end{aligned}
$$

In order to get rid of the first integral at the right-hand side of inequality (26), we transfer it to the left to obtain

$$
\begin{aligned}
& \frac{1}{2} \int_{Q_{T}} \lambda^{4} \tau^{4} \rho^{2 s+3}|\varphi|^{2} e^{-2 \alpha} d x d t \\
& -C \tau \int_{Q_{T}}\left(\tau \rho+\|a\|_{L^{\infty}\left(Q_{T}\right)}^{2}\right) \rho^{2 s}|\varphi|^{2} e^{-2 \alpha} d x d t \\
= & \int_{Q_{T}}\left(\frac{1}{2} \lambda^{4} \tau^{3} \rho^{3}-C \tau \rho-C\|a\|_{L^{\infty}\left(Q_{T}\right)}^{2}\right) \tau \rho^{2 s}|\varphi|^{2} e^{-2 \alpha} d x d t \\
\geq & \int_{Q_{T}}\left(\frac{1}{4} \lambda^{4} \tau^{3} \rho^{3}-C\|a\|_{L^{\infty}\left(Q_{T}\right)}^{2}\right) \tau \rho^{2 s}|\varphi|^{2} e^{-2 \alpha} d x d t,
\end{aligned}
$$

provided (see (16)) $\tau \geq C \frac{T^{2}}{\lambda^{2}}$.

Now notice that

$$
\frac{1}{4} \lambda^{4} \tau^{3} \rho^{3}-C\|a\|_{L^{\infty}\left(Q_{T}\right)}^{2} \geq 2^{4} \frac{\lambda^{4}}{T^{6}} \tau^{3}-C\|a\|_{L^{\infty}\left(Q_{T}\right)}^{2} \geq 0
$$

provided

$$
\begin{aligned}
\tau & \geq C \frac{T^{2}}{(2 \lambda)^{4 / 3}}\|a\|_{L^{\infty}\left(Q_{T}\right)}^{2 / 3} \\
& \geq C T^{2}\|a\|_{L^{\infty}\left(Q_{T}\right)}^{2 / 3} .
\end{aligned}
$$

Taking into account these computations, (26) becomes

$$
I(s, \varphi) \leq C \lambda^{4} \int_{0}^{T} \int_{\omega^{\prime}}\left(\varphi^{2} \delta^{2 s+3}+w^{2} \delta^{2 s+4}\right) e^{-2 \alpha} d x d t
$$

provided $\tau \geq \tau_{1}$.

Similarly, for the second equation of problem (6), we obtain for $s \geq-3 / 2$ and using (29) 


$$
\begin{aligned}
I(s, w) & \leq C\left(\int_{Q_{T}} \varphi^{2} \delta^{2 s} e^{-2 \alpha} d x d t+\lambda^{4} \int_{0}^{T} \int_{\omega^{\prime}} w^{2} \delta^{2 s+3} e^{-2 \alpha} d x d t\right) \\
& \leq C\left(\frac{1}{\lambda^{4}} I\left(s-\frac{3}{2}, \varphi\right)+\lambda^{4} \int_{0}^{T} \int_{\omega^{\prime}} w^{2} \delta^{2 s+3} e^{-2 \alpha} d x d t\right) \\
& \leq C \int_{0}^{T} \int_{\omega^{\prime}}\left(\varphi^{2} \delta^{2 s}+w^{2} \delta^{2 s+1}+\lambda^{4} w^{2} \delta^{2 s+3}\right) e^{-2 \alpha} d x d t \\
& \leq C \int_{0}^{T} \int_{\omega^{\prime}}\left(\varphi^{2} \delta^{2 s}+\lambda^{4} w^{2} \delta^{2 s+3}\right) e^{-2 \alpha} d x d t
\end{aligned}
$$

the last inequality being obtained by noting that $\delta \geq 1$ for $\tau$ sufficiently large.

Adding up inequalities (29) and (30), we get (21).

The consequence (22) follows from $\delta \geq 1$ for $\tau$ sufficiently large and by taking $s=-\frac{3}{2}$

We are now ready to state our crucial lemma.

Lemma 3.4. Under the assumptions of Lemma 3.2, $\forall r \in[0,2)$ there exists a constant $C=C_{r}$ such that

$$
\int_{0}^{T} \int_{\omega^{\prime}}\left(\varphi^{2}+w^{2}\right) e^{-2 \alpha} d x d t \leq C\left(1+\frac{\tau^{8}}{T^{14}}+\|a\|_{L^{\infty}\left(Q_{T}\right)}^{6}\right) \int_{0}^{T} \int_{\omega} e^{-r \alpha} w^{2} d x d t .
$$

As an immediate consequence, it follows that

$$
I(-3, \varphi)+I\left(-\frac{3}{2}, w\right) \leq C\left(1+\frac{\tau^{8}}{T^{14}}+\|a\|_{L^{\infty}\left(Q_{T}\right)}^{6}\right) \int_{0}^{T} \int_{\omega} e^{-r \alpha} w^{2} d x d t
$$

Remark 3.5. We will see later that it is important to be able to choose $r>1$. Notice that $r$ cannot be equal to 2 : this is the "cost" of the control by a single force.

Proof. The main idea is to estimate $\int_{0}^{T} \int_{\omega^{\prime}} \varphi^{2} e^{-2 \alpha} d x d t$ by $\int_{0}^{T} \int_{\omega} e^{-r \alpha} w^{2} d x d t$ for some $r \in[0,2)$ using the second equation of (19). To do this, we first localize the system in space, multiply the second equation by $-\beta_{0} e^{-2 \alpha} \eta \varphi$, and manage the "bad" terms appearing (see $\Lambda(t)$ in (34) and our paper [2] for the construction of this function in an abstract setting).

Let $\xi \in C^{\infty}\left(R^{n}\right)$ be a truncation function satisfying

$$
\begin{cases}\xi(x)=1 & \forall x \in \omega^{\prime}, \\ 0<\xi(x) \leq 1 & \forall x \in \omega^{\prime \prime} \\ \xi(x)=0 & \forall x \in \mathbb{R}^{n} \backslash \omega^{\prime \prime}\end{cases}
$$

where $\omega^{\prime} \Subset \omega^{\prime \prime} \Subset \omega \Subset \Omega$. We introduce the function $\eta:=\xi^{6}$. For real numbers $\beta_{0}, \beta_{1}, p, q>0$, which will be chosen below, set

$$
\Lambda(t)=\int_{\Omega}\left(e^{-p \alpha} \eta^{4 / 3} \varphi^{2}-\beta_{0} e^{-2 \alpha} \eta \varphi w+\beta_{1} e^{-q \alpha} \eta^{2 / 3} w^{2}\right) d x
$$

Differentiating $\Lambda$ with respect to $t$ and replacing $\varphi_{t}$ and $w_{t}$ by their expressions given 
by (19) we obtain

$$
\begin{aligned}
\Lambda^{\prime}= & \int_{\Omega}\left(-p e^{-p \alpha} \eta^{4 / 3} \varphi^{2}+2 \beta_{0} e^{-2 \alpha} \eta \varphi w-\beta_{1} q e^{-q \alpha} \eta^{2 / 3} w^{2}\right) \alpha_{t} d x \\
& +\int_{\Omega} 2 e^{-p \alpha} \eta^{4 / 3} \varphi(\Delta \varphi+a \varphi-\Delta w) d x \\
& -\beta_{0} \int_{\Omega} e^{-2 \alpha} \eta[\varphi(\Delta w+\varphi)+w(\Delta \varphi+a \varphi-\Delta w)] d x \\
& +\int_{\Omega} 2 \beta_{1} e^{-q \alpha} \eta^{2 / 3} w(\Delta w+\varphi) d x .
\end{aligned}
$$

We have $\Lambda(0)=\Lambda(T)=0$, and therefore the integration of $(35)$ over $(0, T)$ yields

$$
\begin{aligned}
\beta_{0} \int_{Q_{T}} e^{-2 \alpha} \eta \varphi^{2} d x= & \int_{Q_{T}}\left\{\left(-p \alpha_{t}+2 a\right) e^{-p \alpha} \eta^{4 / 3} \varphi^{2}-\beta_{1} q \alpha_{t} e^{-q \alpha} \eta^{2 / 3} w^{2}\right. \\
& \left.+\left(\beta_{0}\left(2 \alpha_{t}-a\right) e^{-2 \alpha} \eta+2 \beta_{1} e^{-q \alpha} \eta^{2 / 3}\right) \varphi w\right\} d x d t \\
& +2 \int_{Q_{T}} e^{-p \alpha} \eta^{4 / 3} \varphi \Delta \varphi d x d t \\
& -\int_{Q_{T}}\left(2 e^{-p \alpha} \eta^{4 / 3}+\beta_{0} e^{-2 \alpha} \eta\right) \varphi \Delta w d x d t \\
& -\int_{Q_{T}} \beta_{0} e^{-2 \alpha} \eta w \Delta \varphi d x d t \\
& +\int_{Q_{T}}\left(\beta_{0} e^{-2 \alpha} \eta+2 \beta_{1} e^{-q \alpha} \eta^{2 / 3}\right) w \Delta w d x d t \\
= & J_{1}+J_{2}+J_{3}+J_{4}+J_{5} .
\end{aligned}
$$

Now we estimate each of the five terms $J_{1}, \ldots, J_{5}$.

For $J_{1}$, one has

$$
\begin{aligned}
J_{1}=\int_{Q_{T}} & \left\{\left(-p \alpha_{t}+2 a\right) e^{-p \alpha} \eta^{4 / 3} \varphi^{2}-\beta_{1} q \alpha_{t} e^{-q \alpha} \eta^{2 / 3} w^{2}\right. \\
+ & \left.\left(\beta_{0}\left(2 \alpha_{t}-a\right) e^{-2 \alpha} \eta+2 \beta_{1} e^{-q \alpha} \eta^{2 / 3}\right) \varphi w\right\} d x d t .
\end{aligned}
$$

In order to estimate $\beta_{0}\left(2 \alpha_{t}-a\right) e^{-2 \alpha} \eta \varphi w$ in terms of $e^{-2 \alpha} \eta \varphi^{2}$, we need to bound $\left(2 \alpha_{t}-a\right)^{2} e^{-2 \alpha} \eta^{2 / 3} w^{2}$. So, since $\alpha_{t} \notin L^{\infty}\left(Q_{T}\right)$, we introduce $r \in[0,2)$ and write $e^{-2 \alpha}=e^{-(2-r) \alpha} e^{-r \alpha}$. Assuming that

$$
p>2, q>1+\frac{r}{2},
$$

we get, with $\beta_{1} \geq 1$,

$$
\begin{aligned}
J_{1} \leq & \left(\frac{1}{2}+\left\|\left(-p \alpha_{t}+2 a\right) e^{-(p-2) \alpha} \eta^{1 / 3}\right\|_{L^{\infty}\left(Q_{T}\right)}\right) \int_{Q_{T}} e^{-2 \alpha} \eta \varphi^{2} d x d t \\
& +\left\{\frac{1}{2}\left\|\beta_{0}\left(2 \alpha_{t}-a\right) e^{-\left(1-\frac{r}{2}\right) \alpha} \eta^{1 / 3}+2 \beta_{1} e^{-\left(q-1-\frac{r}{2}\right) \alpha}\right\|_{L^{\infty}\left(Q_{T}\right)}^{2}\right. \\
& \left.+\beta_{1} q\left\|\alpha_{t} e^{-(q-r) \alpha} \eta^{1 / 3}\right\|_{L^{\infty}\left(Q_{T}\right)}\right\} \int_{Q_{T}} \eta^{1 / 3} e^{-r \alpha} w^{2} d x d t . \\
\leq & C\left[\left(1+\left\|\alpha_{t} e^{-(p-2) \alpha}\right\|_{L^{\infty}\left(Q_{T}\right)}+\|a\|_{L^{\infty}\left(Q_{T}\right)}^{2}\right) \int_{Q_{T}} e^{-2 \alpha} \eta \varphi^{2} d x d t\right.
\end{aligned}
$$




$$
\begin{aligned}
& +\left(\left(\|a\|_{L^{\infty}\left(Q_{T}\right)}^{2}+\left\|\alpha_{t} e^{-\left(1-\frac{r}{2}\right) \alpha}\right\|_{L^{\infty}\left(Q_{T}\right)}\right) \beta_{0}^{2}\right. \\
& \left.\left.+\left(1+\left\|\alpha_{t} e^{-(q-r) \alpha}\right\|_{L^{\infty}\left(Q_{T}\right)}\right) \beta_{1}^{2}\right) \int_{Q_{T}} \eta^{1 / 3} e^{-r \alpha} w^{2} d x d t\right]
\end{aligned}
$$

where $C=C\left(p, q,\|\eta\|_{L^{\infty}(\Omega)}\right)$. Now, assuming that $\tau \geq \tau_{1}$ and $\beta_{1} \geq 1$, one obtains

$$
\begin{aligned}
J_{1} \leq & C\left[\left(1+\frac{\tau}{T^{3}}+\|a\|_{L^{\infty}\left(Q_{T}\right)}^{2}\right) \int_{Q_{T}} e^{-2 \alpha} \eta \varphi^{2} d x d t\right. \\
& \left.+\left(1+\|a\|_{L^{\infty}\left(Q_{T}\right)}^{2}+\frac{\tau^{2}}{T^{6}}\right)\left(\beta_{1}^{2}+\beta_{0}^{2}\right) \int_{Q_{T}} \eta^{1 / 3} e^{-r \alpha} w^{2} d x d t\right] .
\end{aligned}
$$

Concerning $J_{2}$,

$$
\begin{aligned}
J_{2}= & 2 \int_{Q_{T}} e^{-p \alpha} \eta^{4 / 3} \varphi \Delta \varphi d x d t \\
= & -2 \int_{Q_{T}} e^{-p \alpha} \eta^{4 / 3}|\nabla \varphi|^{2} d x d t-2 \int_{Q_{T}} \varphi \nabla \varphi \cdot \nabla\left(e^{-p \alpha} \eta^{4 / 3}\right) d x d t \\
= & -2 \int_{Q_{T}} e^{-p \alpha} \eta^{4 / 3}|\nabla \varphi|^{2} d x d t+\int_{Q_{T}} \varphi^{2} \cdot \Delta\left(e^{-p \alpha} \eta^{4 / 3}\right) d x d t \\
= & -2 \int_{Q_{T}} e^{-p \alpha} \eta^{4 / 3}|\nabla \varphi|^{2} d x d t \\
& +\int_{Q_{T}}\left(e^{-2 \alpha} \eta \varphi^{2}\right) \cdot e^{2 \alpha} \eta^{-1} \Delta\left(e^{-p \alpha} \eta^{4 / 3}\right) d x d t .
\end{aligned}
$$

We have

$$
\begin{aligned}
\Delta\left(e^{-p \alpha} \eta^{l}\right)= & e^{-p \alpha}\left[\left(p^{2}|\nabla \alpha|^{2}-p \Delta \alpha\right) \eta^{l}+l(\Delta \eta-2 p \nabla \alpha . \nabla \eta) \eta^{l-1}\right. \\
& \left.+p(p-1)|\nabla \eta|^{2} \eta^{l-2}\right] .
\end{aligned}
$$

So

$$
\begin{aligned}
e^{2 \alpha} \eta^{-1} \Delta\left(e^{-p \alpha} \eta^{4 / 3}\right)= & e^{-(p-2) \alpha}\left[\left(p^{2}|\nabla \alpha|^{2}-p \Delta \alpha\right) \eta^{1 / 3}\right. \\
& +\frac{4}{3}(\Delta \eta-2 p \nabla \alpha . \nabla \eta) \eta^{-2 / 3} \\
& \left.+p(p-1)|\nabla \eta|^{2} \eta^{-5 / 3}\right]
\end{aligned}
$$

Note that

$$
\begin{aligned}
& \frac{\nabla \eta}{\eta^{5 / 6}}=6 \nabla \xi \in L^{\infty}(\Omega), \quad \frac{\nabla \eta}{\eta^{2 / 3}}=6 \xi \nabla \xi \in L^{\infty}(\Omega), \\
& \frac{\Delta \eta}{\eta^{2 / 3}}=30|\nabla \xi|^{2}+6 \xi \Delta \xi \in L^{\infty}(\Omega) .
\end{aligned}
$$

It follows from this last computation and $p>2$ that

$$
\left\|e^{2 \alpha} \eta^{-1} \Delta\left(e^{-p \alpha} \eta^{4 / 3}\right)\right\|_{L^{\infty}\left(Q_{T}\right)} \leq C\left(1+\frac{\tau^{2}}{T^{4}}\right)
$$


where $C=C\left(p,\|\eta\|_{L^{\infty}(\Omega)}\right)$. Coming back to $J_{2}$, we get

$$
J_{2} \leq-2 \int_{Q_{T}} e^{-p \alpha} \eta^{4 / 3}|\nabla \varphi|^{2} d x d t+C\left(1+\frac{\tau^{2}}{T^{4}}\right) \int_{Q_{T}} e^{-2 \alpha} \eta \varphi^{2} d x d t .
$$

We now estimate $J_{3}+J_{4}$. We have

$$
\begin{aligned}
J_{3}+J_{4}= & -\int_{Q_{T}} \beta_{0} e^{-2 \alpha} \eta(\varphi \Delta w+w \Delta \varphi) d x d t-\int_{Q_{T}} 2 e^{-p \alpha} \eta^{4 / 3} \varphi \Delta w d x d t \\
= & -\int_{Q_{T}} \beta_{0} e^{-2 \alpha} \eta(\Delta(\varphi w)-2 \nabla \varphi \cdot \nabla w) d x d t \\
& +\int_{Q_{T}} 2 e^{-p \alpha} \eta^{4 / 3} \nabla \varphi \cdot \nabla w d x d t \\
& +\int_{Q_{T}} 2 \varphi \nabla\left(e^{-p \alpha} \eta^{4 / 3}\right) \cdot \nabla w d x d t \\
= & -\beta_{0} \int_{Q_{T}} \Delta\left(e^{-2 \alpha} \eta\right) \varphi w d x d t \\
& +2 \int_{Q_{T}}\left(\beta_{0} e^{-2 \alpha} \eta+e^{-p \alpha} \eta^{4 / 3}\right) \nabla \varphi \cdot \nabla w d x d t \\
& +\int_{Q_{T}} 2 \varphi \nabla\left(e^{-p \alpha} \eta^{4 / 3}\right) \cdot \nabla w d x d t .
\end{aligned}
$$

Proceeding as previously, thanks to the assumption $r<2$, we first get

$$
\begin{aligned}
\left|\beta_{0} \int_{Q_{T}} \Delta\left(e^{-2 \alpha} \eta\right) \varphi w d x d t\right| \leq & C\left(\int_{Q_{T}} e^{-2 \alpha} \eta \varphi^{2} d x d t\right. \\
& \left.+\beta_{0}^{2}\left(1+\frac{\tau^{4}}{T^{8}}\right) \int_{Q_{T}} \eta^{1 / 3} e^{-r \alpha} w^{2} d x d t\right) .
\end{aligned}
$$

In the same way, with $p>2$ and $\eta^{-1 / 6} \nabla \eta \in L^{\infty}(\Omega)$,

$$
\begin{aligned}
\left|\int_{Q_{T}} 2 \varphi \nabla\left(e^{-p \alpha} \eta^{4 / 3}\right) . \nabla w d x d t\right| \leq & C\left(\int_{Q_{T}} e^{-2 \alpha} \eta \varphi^{2} d x d t\right. \\
& \left.+\left(1+\frac{\tau^{2}}{T^{4}}\right) \int_{Q_{T}} \eta^{2 / 3} e^{-2(p-1) \alpha}|\nabla w|^{2} d x d t\right) .
\end{aligned}
$$

It appears that

$$
\begin{aligned}
J_{3}+J_{4} \leq & C\left(\int_{Q_{T}} e^{-2 \alpha} \eta \varphi^{2} d x d t+\beta_{0}^{2}\left(1+\frac{\tau^{4}}{T^{8}}\right) \int_{Q_{T}} \eta^{1 / 3} e^{-r \alpha} w^{2} d x d t\right. \\
& \left.+\left(1+\frac{\tau^{2}}{T^{4}}\right) \int_{Q_{T}} \eta^{2 / 3} e^{-2(p-1) \alpha}|\nabla w|^{2} d x d t\right) \\
& +2 \int_{Q_{T}}\left(\beta_{0} e^{-2 \alpha} \eta+e^{-p \alpha} \eta^{4 / 3}\right) \nabla \varphi \cdot \nabla w d x d t .
\end{aligned}
$$

Finally, we estimate $J_{5}$.

$$
J_{5}=\int_{Q_{T}}\left(\beta_{0} e^{-2 \alpha} \eta+2 \beta_{1} e^{-q \alpha} \eta^{2 / 3}\right) w \Delta w d x d t
$$




$$
\begin{aligned}
= & -\int_{Q_{T}}\left(\beta_{0} e^{-2 \alpha} \eta+2 \beta_{1} e^{-q \alpha} \eta^{2 / 3}\right)|\nabla w|^{2} d x d t \\
& +\frac{1}{2} \int_{Q_{T}} \Delta\left(\beta_{0} e^{-2 \alpha} \eta+2 \beta_{1} e^{-q \alpha} \eta^{2 / 3}\right) w^{2} d x d t .
\end{aligned}
$$

Again, in the same way, we get using the condition $q>r$ and the definition of $\eta$ :

$\frac{1}{2}\left|\int_{Q_{T}} \Delta\left(\beta_{0} e^{-2 \alpha} \eta+2 \beta_{1} e^{-q \alpha} \eta^{2 / 3}\right) w^{2} d x d t\right| \leq C \beta_{1}\left(1+\frac{\tau^{2}}{T^{4}}\right) \int_{Q_{T}} \eta^{1 / 3} e^{-r \alpha} w^{2} d x d t$.

Thus

$$
\begin{aligned}
J_{5} \leq & -\int_{Q_{T}}\left(\beta_{0} e^{-2 \alpha} \eta+2 \beta_{1} e^{-q \alpha} \eta^{2 / 3}\right)|\nabla w|^{2} d x d t \\
& +C \beta_{1}\left(1+\frac{\tau^{2}}{T^{4}}\right) \int_{Q_{T}} \eta^{1 / 3} e^{-r \alpha} w^{2} d x d t .
\end{aligned}
$$

Fix $\beta_{0}=2 C\left(1+\frac{\tau^{2}}{T^{4}}+\|a\|_{L^{\infty}\left(Q_{T}\right)}^{2}\right)$. Then from (38), (39), (40), and (41), we get with $\tau \geq \tau_{0}$, conditions (37) and $\beta_{1} \geq 1$ :

$$
\begin{aligned}
\int_{Q_{T}} e^{-2 \alpha} \eta \varphi^{2} d x \leq & 2 C\left(1+\|a\|_{L^{\infty}\left(Q_{T}\right)}^{2}+\frac{\tau^{4}}{T^{8}}\right) \frac{\left(\beta_{1}^{2}+\beta_{0}^{2}\right)}{\beta_{0}} \int_{Q_{T}} \eta^{1 / 3} e^{-r \alpha} w^{2} d x d t \\
& -\frac{4}{\beta_{0}} \int_{Q_{T}} e^{-p \alpha} \eta^{4 / 3}|\nabla \varphi|^{2} d x d t \\
& -\frac{2}{\beta_{0}} \int_{Q_{T}}\left(\beta_{0} e^{-2 \alpha} \eta+2 \beta_{1} e^{-q \alpha} \eta^{2 / 3}\right. \\
& \left.-C\left(1+\frac{\tau^{2}}{T^{4}}\right) \eta^{2 / 3} e^{-2(p-1) \alpha}\right)|\nabla w|^{2} d x d t \\
& +\frac{4}{\beta_{0}} \int_{Q_{T}}\left(\beta_{0} e^{-2 \alpha} \eta+e^{-p \alpha} \eta^{4 / 3}\right) \nabla \varphi \cdot \nabla w d x d t .
\end{aligned}
$$

Consider the three last terms in the right-hand side of the previous inequality. Assume moreover that

$$
\frac{1}{2} q+1<p<4-q .
$$

For $\beta_{1}$ sufficiently large, we have

$$
\begin{aligned}
\left(\beta_{0} e^{-2 \alpha} \eta+e^{-p \alpha} \eta^{4 / 3}\right)^{2} \leq & 2 e^{-p \alpha} \eta^{4 / 3}\left(\beta_{0} e^{-2 \alpha} \eta+2 \beta_{1} e^{-q \alpha} \eta^{2 / 3}\right. \\
& \left.-C\left(1+\frac{\tau^{2}}{T^{4}}\right) \eta^{2 / 3} e^{-2(p-1) \alpha}\right) \text { on } Q_{T} .
\end{aligned}
$$

Here is the proof. Indeed, it is sufficient to prove

$$
\frac{1}{4}\left(\beta_{0} e^{-\left(2-\frac{p+q}{2}\right) \alpha}+e^{-\frac{p-q}{2} \alpha} \eta^{1 / 3}\right)^{2}+\frac{1}{2} C\left(1+\frac{\tau^{2}}{T^{4}}\right) e^{-(2 p-q-2) \alpha} \leq \beta_{1} \text { on } Q_{T} .
$$

Taking into account (42), this last estimate is true if, for instance,

$$
\beta_{1} \geq C\left(\left(\beta_{0}+1\right)^{2}+1+\frac{\tau^{2}}{T^{4}}\right)
$$


with a large constant $C=C\left(\Omega, \omega, \omega^{\prime}, \eta\right)$. To summarize, we have

$$
\int_{Q_{T}} e^{-2 \alpha} \eta \varphi^{2} d x d t \leq C \frac{\left(1+\|a\|_{L^{\infty}\left(Q_{T}\right)}^{2}+\frac{\tau^{4}}{T^{8}}\right)\left(\beta_{1}^{2}+\beta_{0}^{2}\right)}{\beta_{0}} \int_{Q_{T}} \eta^{1 / 3} e^{-r \alpha} w^{2} d x d t,
$$

and all the computations are valid with the following conditions:

$$
\begin{aligned}
r & <2, p>2, q>1+\frac{r}{2}, \frac{1}{2} q+1<p<4-q, \\
\beta_{0} & =2 C\left(1+\frac{\tau^{2}}{T^{4}}+\|a\|_{L^{\infty}\left(Q_{T}\right)}^{2}\right), \\
\beta_{1} & \geq C\left(\left(\beta_{0}+1\right)^{2}+1+\frac{\tau^{2}}{T^{4}}\right), \\
\tau & \geq \tau_{1} .
\end{aligned}
$$

It is clear that there is a nonempty set of $(p, q, r)$ verifying (43): for instance, $\left(2+\frac{1}{16}, 2-\frac{1}{8}, \frac{3}{2}\right)$ satisfies this condition. With maybe a modified constant $C$, we get the following final estimate:

$$
\int_{Q_{T, \omega^{\prime}}} e^{-2 \alpha} \varphi^{2} d x d t \leq C\left(1+\frac{\tau^{8}}{T^{14}}+\|a\|_{L^{\infty}\left(Q_{T}\right)}^{6}\right) \int_{Q_{T}, \omega} e^{-r \alpha} w^{2} d x d t
$$

where $Q_{T, \omega}=(0, T) \times \omega$. This final estimate ends the proof of the lemma.

4. Proof of Lemma 2.3. For $r \in(1,2)$ and $\varepsilon>0$, we define

$$
J_{\varepsilon}(g)=\frac{1}{2} \int_{Q_{T}} e^{r \alpha} g^{2} d x d t+\frac{1}{2 \varepsilon}\|(\psi, v)(T)\|_{L^{2}(\Omega)}^{2},
$$

where $g \in L^{2}\left(Q_{T}\right)$ and $(\psi, v)$ is the associated solution of (6). Introduce also the dual functional (see [11]):

$$
J_{\varepsilon}^{*}\left(Y_{0}\right)=\frac{1}{2} \int_{0}^{T} \int_{\omega} e^{-r \alpha} w^{2} d x d t+\frac{\varepsilon}{2}\left\|Y_{0}\right\|_{L^{2}(\Omega)}^{2}+\int_{\Omega} Y(0) \cdot X_{0} d x,
$$

where $X_{0}=\left(\psi_{0}, v_{0}\right) \in H_{0}^{1}(\Omega) \times H_{0}^{1}(\Omega)$ is the fixed initial data of $(6)$ and $Y=(\varphi, w)$ is the solution of the backward linear system with initial data $Y_{0}=\left(\varphi_{0}, w_{0}\right) \in L^{2}(\Omega) \times$ $L^{2}(\Omega)$

$$
\begin{cases}-\varphi_{t}=\Delta \varphi+a \varphi-\Delta w & \text { in }(0, T) \times \Omega=Q_{T} \\ -w_{t}=\Delta w+\varphi & \text { in } Q_{T} \\ \varphi=w=0 & \text { on }(0, T) \times \partial \Omega=\Sigma_{T} \\ \varphi(T)=\varphi_{0}, w(T)=w_{0}, & \text { in } \Omega\end{cases}
$$

It is easy to prove that the minimization problems $\min _{g} J_{\varepsilon}(g)$ and $\min _{Y_{0}} J_{\varepsilon}^{*}\left(Y_{0}\right)$ have both exactly one solution $\left(g_{\varepsilon}, Y_{0 \varepsilon}\right)$ and, moreover, by the maximum principle (or see, for instance, [11])

$$
g_{\varepsilon}=\chi_{\omega} e^{-r \alpha} w_{\varepsilon} \text { on } Q_{T} ; Y_{0 \varepsilon}=-\frac{1}{\varepsilon}\left(\psi_{\varepsilon}, v_{\varepsilon}\right)(T) \text { on } \Omega,
$$


where $\left(\psi_{\varepsilon}, v_{\varepsilon}\right)$ (resp., $\left.\left(\varphi_{\varepsilon}, w_{\varepsilon}\right)\right)$ is the solution of $(6)$ (resp., (44)) associated with $g_{\varepsilon}$ (resp., $\left.Y_{0 \varepsilon}\right)$. Since $J_{\varepsilon}^{*}\left(Y_{0 \varepsilon}\right) \leq 0$, we get

$$
\frac{1}{2} \int_{0}^{T} \int_{\omega} e^{-r \alpha} w_{\varepsilon}^{2} d x d t+\frac{1}{2 \varepsilon}\left\|\left(\psi_{\varepsilon}, v_{\varepsilon}\right)(T)\right\|_{L^{2}(\Omega)}^{2} \leq\left\|\left(\varphi_{\varepsilon}, w_{\varepsilon}\right)(0)\right\|_{L^{2}(\Omega)} \cdot\left\|X_{0}\right\|_{L^{2}(\Omega)} .
$$

To obtain a uniform estimate, we will need the following result.

Lemma 4.1. For $r \in(0,2)$, any solution pair of (44) satisfies the estimate

$$
\|(\varphi, w)(0)\|_{L^{2}(\Omega)}^{2} \leq C_{T} \int_{0}^{T} \int_{\omega} e^{-r \alpha} w^{2} d x d t
$$

with

$$
C_{T}=\exp \left(C\left(1+\frac{1}{T}+\left(1+\|a\|_{L^{\infty}\left(Q_{T}\right)}\right) T+\|a\|_{L^{\infty}\left(Q_{T}\right)}^{2 / 3}\right)\right) .
$$
$\forall \varepsilon>0$

We will prove this lemma at the end of this section. From (47) and (47), we get

$$
\frac{1}{2} \int_{0}^{T} \int_{\omega} e^{-r \alpha} w_{\varepsilon}^{2} d x d t+\frac{1}{2 \varepsilon}\left\|\left(\psi_{\varepsilon}, v_{\varepsilon}\right)(T)\right\|_{L^{2}(\Omega)}^{2} \leq C_{T}\left\|X_{0}\right\|_{L^{2}(\Omega)}^{2} .
$$

We obtain from this last estimate a control in $L^{2}\left(Q_{T}\right)$; however, as it appeared clearly in the proof of Theorem 2.1, it is not sufficient. So we will prove that our control is in $L^{q_{N}}\left(Q_{T}\right)$.

We introduce $\zeta_{\varepsilon}=e^{-r \alpha} w_{\varepsilon}$. It satisfies by (44)

$$
\begin{cases}\left(\zeta_{\varepsilon}\right)_{t}+\Delta \zeta_{\varepsilon}=f_{\varepsilon} & \text { in }(0, T) \times \Omega=Q_{T} \\ \zeta_{\varepsilon}=0 & \text { on }(0, T) \times \partial \Omega=\Sigma_{T} \\ \zeta_{\varepsilon}(T)=0 & \text { in } \Omega,\end{cases}
$$

with

$$
f_{\varepsilon}=-2 r \nabla \alpha \cdot\left(e^{-r \alpha} \nabla w_{\varepsilon}\right)+\left(\Delta\left(e^{-r \alpha}\right)-\left(e^{-r \alpha}\right)_{t}\right) w_{\varepsilon}-e^{-r \alpha} \varphi_{\varepsilon} .
$$

By parabolic regularity, we have

$$
\left\|\zeta_{\varepsilon}\right\|_{W_{2}^{2,1}\left(Q_{T}\right)} \leq C\left\|f_{\varepsilon}\right\|_{L^{2}\left(Q_{T}\right)} .
$$

On the other hand, setting

$$
I_{1}=\int_{Q_{T}} e^{-2 r \alpha} \varphi_{\varepsilon}^{2} d x d t
$$

we have, using (32) in Lemma 3.4,

$$
\begin{aligned}
I_{1} & =\int_{Q_{T}}\left(\delta^{3} e^{-2(r-1) \alpha}\right)\left(\delta^{-3} e^{-2 \alpha} \varphi_{\varepsilon}^{2}\right) d x d t \\
& \leq\left\|\delta^{3} e^{-2(r-1) \alpha}\right\|_{L^{\infty}\left(Q_{T}\right)} \int_{Q_{T}} \delta^{-3} e^{-2 \alpha} \varphi_{\varepsilon}^{2} d x d t \\
& \leq C\left\|\delta^{3} e^{-2(r-1) \alpha}\right\|_{L^{\infty}\left(Q_{T}\right)}\left(1+\frac{\tau^{8}}{T^{14}}+\|a\|_{L^{\infty}\left(Q_{T}\right)}^{6}\right) \int_{0}^{T} \int_{\omega} e^{-r \alpha} w_{\varepsilon}^{2} d x d t \\
& \leq C\left(1+\frac{\tau^{8}}{T^{14}}+\|a\|_{L^{\infty}\left(Q_{T}\right)}^{6}\right) \int_{0}^{T} \int_{\omega} e^{-r \alpha} w_{\varepsilon}^{2} d x d t .
\end{aligned}
$$


Notice that $\left\|\delta^{3} e^{-2(r-1) \alpha}\right\|_{L^{\infty}\left(Q_{T}\right)}$ is finite since we have assumed $r>1$ and the same remark holds in what follows. In the same way, setting

$$
\begin{gathered}
I_{2}=\int_{Q_{T}}\left|\nabla \alpha \cdot\left(e^{-r \alpha} \nabla w_{\varepsilon}\right)\right|^{2} d x d t \\
I_{2} \leq \int_{Q_{T}}\left(|\nabla \alpha|^{2} \delta^{2} e^{-2(r-1) \alpha}\right)\left(\delta^{-2} e^{-2 \alpha}\left|\nabla w_{\varepsilon}\right|^{2}\right) d x d t \\
\leq\left\||\nabla \alpha|^{2} \delta^{2} e^{-2(r-1) \alpha}\right\|_{L^{\infty}\left(Q_{T}\right)} C\left(1+\frac{\tau^{8}}{T^{14}}+\|a\|_{L^{\infty}\left(Q_{T}\right)}^{6}\right) \int_{0}^{T} \int_{\omega} e^{-r \alpha} w_{\varepsilon}^{2} d x d t \\
\leq C\left(1+\frac{\tau^{8}}{T^{14}}+\|a\|_{L^{\infty}\left(Q_{T}\right)}^{6}\right) \int_{0}^{T} \int_{\omega} e^{-r \alpha} w_{\varepsilon}^{2} d x d t,
\end{gathered}
$$

and, if

$$
\begin{gathered}
I_{3}=\int_{Q_{T}}\left|\left(\Delta\left(e^{-r \alpha}\right)-\left(e^{-r \alpha}\right)_{t}\right) w_{\varepsilon}\right|^{2} d x d t \\
I_{3}=\left.\int_{Q_{T}}\left|-r \Delta \alpha+r^{2}\right| \nabla \alpha\right|^{2}+\left.r \alpha_{t}\right|^{2} e^{-2 r \alpha} w_{\varepsilon}^{2} d x d t \\
=\int_{Q_{T}}\left(\left.\left|-r \Delta \alpha+r^{2}\right| \nabla \alpha\right|^{2}+\left.r \alpha_{t}\right|^{2} e^{-2(r-1) \alpha}\right)\left(e^{-2 \alpha} w_{\varepsilon}^{2}\right) d x d t \\
\leq C\left\|\left.\left|-r \Delta \alpha+r^{2}\right| \nabla \alpha\right|^{2}+\left.r \alpha_{t}\right|^{2} e^{-2(r-1) \alpha}\right\|_{L^{\infty}\left(Q_{T}\right)} \\
\\
\quad\left(1+\frac{\tau^{8}}{T^{14}}+\|a\|_{L^{\infty}\left(Q_{T}\right)}^{6}\right) \int_{0}^{T} \int_{\omega} e^{-r \alpha} w_{\varepsilon}^{2} d x d t \\
\leq C\left(1+\frac{\tau^{8}}{T^{14}}+\|a\|_{L^{\infty}\left(Q_{T}\right)}^{6}\right) \int_{0}^{T} \int_{\omega} e^{-r \alpha} w_{\varepsilon}^{2} d x d t .
\end{gathered}
$$

It follows from these three last inequalities and (49) that

$$
\left\|\zeta_{\varepsilon}\right\|_{W_{2}^{2,1}\left(Q_{T}\right)}^{2} \leq C\left(1+\frac{\tau^{8}}{T^{14}}+\|a\|_{L^{\infty}\left(Q_{T}\right)}^{6}\right) \int_{0}^{T} \int_{\omega} e^{-r \alpha} w_{\varepsilon}^{2} d x d t .
$$

Now, by the embedding $W_{2}^{2,1}\left(Q_{T}\right) \hookrightarrow L^{q_{N}}\left(Q_{T}\right)$ (see, for instance, [18, Lemma 3.2, p. 80])

$$
\left\|\zeta_{\varepsilon}\right\|_{L^{q_{N}\left(Q_{T}\right)}}^{2} \leq C\left(1+\frac{\tau^{8}}{T^{14}}+\|a\|_{L^{\infty}\left(Q_{T}\right)}^{6}\right) \int_{0}^{T} \int_{\omega} e^{-r \alpha} w_{\varepsilon}^{2} d x d t .
$$

Going back to our control, we get, using (48),

$$
\begin{aligned}
\left\|g_{\varepsilon}\right\|_{L^{q_{N}\left(Q_{T}\right)}}^{2} & =\left\|\chi_{\omega} \zeta_{\varepsilon}\right\|_{L^{q_{N}\left(Q_{T}\right)}}^{2} \\
& \leq C\left(1+\frac{\tau^{8}}{T^{14}}+\|a\|_{L^{\infty}\left(Q_{T}\right)}^{6}\right) \int_{0}^{T} \int_{\omega} e^{-r \alpha} w_{\varepsilon}^{2} d x d t \\
& \leq C_{T}\left\|X_{0}\right\|_{L^{2}(\Omega)}^{2} .
\end{aligned}
$$


From (50) and [18, Theorem 10.4, p. 621], it follows, at least for a subsequence, that for $\varepsilon \rightarrow 0$

$$
\begin{array}{rlrl}
g_{\varepsilon} & \rightarrow g & & \text { weakly in } L^{q_{N}}\left(Q_{T}\right), \\
\left(\psi_{\varepsilon}, v_{\varepsilon}\right) & \rightarrow(\psi, v) & \text { weakly in } L^{2}\left(0, T ; H_{0}^{1}(\Omega)\right) \cap W_{q_{N}}^{2,1}\left(Q_{T}\right),
\end{array}
$$

and $((\psi, v), g)$ satisfy $(6)$ with $(\psi, v)(T)=0$ and

$$
\left\|\chi_{\omega} g\right\|_{L^{q_{N}\left(Q_{T}\right)}}^{2} \leq C_{T}\left\|X_{0}\right\|_{L^{2}(\Omega)}^{2} .
$$

To complete the proof, it remains to show Lemma 4.1.

Proof of Lemma 4.1. According to Lemma 3.4

$\int_{T / 4}^{3 T / 4} \int_{\Omega}\left(\delta^{-3}|\varphi|^{2}+|w|^{2}\right) e^{-2 \alpha} d x d t \leq C\left(1+\frac{\tau^{8}}{T^{14}}+\|a\|_{L^{\infty}\left(Q_{T}\right)}^{6}\right) \int_{0}^{T} \int_{\omega} e^{-r \alpha} w^{2} d x d t$

$(51)$

provided $\tau \geq \tau_{1}$. Let us estimate $\delta^{-3} e^{-2 \alpha}$ from below on $(T / 4,3 T / 4) \times \Omega$ :

$$
\begin{aligned}
\delta^{-3}(t, x) e^{-2 \alpha(t, x)} & =\tau^{-3} t^{3}(T-t)^{3} e^{-3 \lambda \beta(x)} \exp \left(-2 \tau \frac{e^{\frac{4}{3} \lambda\|\beta\|_{L} \infty(\Omega)}-e^{\lambda \beta(x)}}{t(T-t)}\right) \\
& \geq \tau^{-3} e^{-3 \lambda\|\beta\|_{L} \infty(\Omega)} t^{3}(T-t)^{3} \exp \left(-2 \tau \frac{e^{\frac{4}{3} \lambda\|\beta\|_{L} \infty(\Omega)}-e^{\lambda \ln 3}}{t(T-t)}\right) \\
& \geq C \tau^{-3} T^{6} \exp \left(-\frac{C \tau}{T^{2}}\right) \\
& \geq C \tau^{-3} \exp \left(-\frac{C \tau}{T^{2}}\right) .
\end{aligned}
$$

Inserting this inequality into (51), we get

$$
\begin{aligned}
& \int_{T / 4}^{3 T / 4} \int_{\Omega}\left(|\varphi|^{2}+|w|^{2}\right) d x d t \\
\leq & C \tau^{3} \exp \left(\frac{C \tau}{T^{2}}\right)\left(1+\frac{\tau^{8}}{T^{14}}+\|a\|_{L^{\infty}\left(Q_{T}\right)}^{6}\right) \int_{0}^{T} \int_{\omega} e^{-r \alpha} w^{2} d x d t .
\end{aligned}
$$

If $(\varphi, w)$ satisfies $(44)$, then for $m=\|a\|_{L^{\infty}\left(Q_{T}\right)}+\frac{1}{2}$ we have

$$
\begin{aligned}
& \frac{d}{d t}\left(e^{-2 m(T-t)}\left(|\varphi(t)|_{2}^{2}+|w(t)|_{2}^{2}\right)\right) \\
& =2 m e^{-2 m(T-t)}\left(|\varphi(t)|_{2}^{2}+|w(t)|_{2}^{2}\right)+2 e^{-2 m(T-t)}\left(\int_{\Omega}\left(w w_{t}+\varphi \varphi_{t}\right)(t) d x\right),
\end{aligned}
$$

or, together with the Cauchy-Schwarz and Young inequalities,

$$
\begin{aligned}
& \frac{d}{d t}\left(e^{-2 m(T-t)}\left(\|\varphi(t)\|_{L^{2}(\Omega)}^{2}+\|w(t)\|_{L^{2}(\Omega)}^{2}\right)\right) \\
& \geq 2 e^{-2 m(T-t)}\left\{\left(m-\frac{1}{2}\right)\|w(t)\|_{L^{2}(\Omega)}^{2}+\left(m-\|a\|_{L^{\infty}\left(Q_{T}\right)}-\frac{1}{2}\right)\|\varphi(t)\|_{L^{2}(\Omega)}^{2}\right\} \geq 0 .
\end{aligned}
$$

The integration of (54) over $[T / 4,3 T / 4]$ yields 


$$
\begin{aligned}
\frac{T}{2} e^{-2 m T}\left(\|\varphi(0)\|_{L^{2}(\Omega)}^{2}+\|w(0)\|_{L^{2}(\Omega)}^{2}\right) & \leq \int_{\frac{T}{4}}^{\frac{3 T}{4}} \int_{\Omega} e^{-2 m(T-t)}\left(\varphi^{2}+w^{2}\right) d x d t \\
& \leq e^{-m \frac{T}{2}} \int_{\frac{T}{4}}^{\frac{3 T}{4}} \int_{\Omega}\left(\varphi^{2}+w^{2}\right) d x d t .
\end{aligned}
$$

Using (52) and setting $\tau=C\left(T+\left(1+\|a\|_{L^{\infty}\left(Q_{T}\right)}^{2 / 3}\right) T^{2}\right)$ with $C$ sufficiently large, we finally arrive at

$$
\begin{aligned}
& \|\varphi(0)\|_{L^{2}(\Omega)}^{2}+\|w(0)\|_{L^{2}(\Omega)}^{2} \\
& \leq \frac{2}{T} e^{m \frac{3 T}{2}} \int_{\frac{T}{4}}^{\frac{3 T}{4}} \int_{\Omega}\left(\varphi^{2}+w^{2}\right) d x d t \\
& \leq \exp \left(C\left(1+\frac{1}{T}+\left(1+\|a\|_{L^{\infty}\left(Q_{T}\right)}\right) T+\|a\|_{L^{\infty}\left(Q_{T}\right)}^{2 / 3}\right)\right) \int_{0}^{T} \int_{\omega} e^{-r \alpha} w^{2} d x d t,
\end{aligned}
$$

which is the desired estimate.

\section{REFERENCES}

[1] F. Ammar Khodja and A. Benabdallah, Sufficient conditions for uniform stabilization of second order equations by dynamical controllers, Dynam. Contin. Discrete Impuls. Systems, 7 (2000), pp. 207-222.

[2] F. Ammar Khodja, A. Benabdallah, C. Dupaix, and I. Kostin, Null-Controllability of Some Systems of Parabolic Type by One Control Force, Prépublications du Laboratoire de Mathématiques de Besançon 2003/08, 2003.

[3] S. Anita And V. Barbu, Local exact controllability of a reaction-diffusion system, Differential Integral Equations, 14 (2001), pp. 577-587.

[4] J. P. Aubin And A. Cellina, Differential Inclusions. Set-Valued Map and Viability Theory, Springer-Verlag, Berlin, 1984.

[5] V. BARBu, Exact controllability of the superlinear heat equation, Appl. Math. Optim., 42 (2000), pp. $73-89$.

[6] V. BARBU, Local controllability of the phase field system, Nonlinear Anal., 50 (2002), pp. 363372.

[7] H. Brezis, Analyse Fonctionnelle. Théorie et Applications, Masson, Paris, 1983.

[8] M. Brokate And J. Sprekels, Hysteresis and Phase Transitions, Appl. Math. Sci. 121, Springer-Verlag, New York, 1996.

[9] G. Caginalp, An analysis of a phase field model for a free boundary, Arch. Rational Mech. Anal., 92 (1986), pp. 205-245.

[10] L. De TeresA, Insensitizing controls for a semilinear heat equation, Comm. Partial Differential Equations, 25 (2000), pp. 39-72.

[11] I. Ekeland and R. Temam, Analyse Convexe et Problèmes Variationnels, Dunod, GauthierVillars, Paris, 1974.

[12] E. FERnÁNDEZ-CARA, Null controllability of the semilinear heat equation, ESAIM Control Optim. Calc. Var., 2 (1997), pp. 87-103.

[13] E. Fernández-CARA And E. ZuAzua, The cost of approximate controllability for heat equations: The linear case, Adv. Differential Equations, 5 (2000), pp. 465-514.

[14] E. Fernández-Cara And E. Zuazua, Null and approximate controllability for weakly blowing up semilinear heat equations, Ann. Inst. H. Poincaré Anal. Non Linéaire, 17 (2000), pp. $583-616$.

[15] A. Funsikov, Optimal Control of Distributed Systems. Theory and Applications. Transl. Math. Monogr., 187, AMS, Providence, RI, 2000.

[16] A. Fursikov and O. Imanuvilov, Controllability of Evolution Equations, Lecture Notes Ser. 34, Seoul National University, Korea, 1996.

[17] O. Yu. Imanuvilov, Boundary controllability of parabolic equations, Uspekhi Mat. Navk, 48 (1993), pp. 211-212 (in Russian); Russian Math. Survey, 48 (1993), pp. 192-194 (in English). 
[18] O. A. Ladyženskaja, V. A. Solonnikov, and N. N. Ural'Ceva, Linear and Quasilinear Equations of Parabolic Type, Transl. Math. Monogr. 23, AMS, Providence, RI, 1968.

[19] J.-L. Lions, Contrôlabilité Exacte, Perturbations et Stabilisation de Systèmes Distribués, Vol. 1, RMA 8, Masson, Paris, 1988.

[20] G. Lebeau and L. Robbiano, Contrôle exact de l'équation de la chaleur, Comm. Partial Differential Equations, 20 (1995), pp. 335-356.

[21] J. ZabcZyk, Mathematical Control Theory: An Introduction, Birkhäuser, Boston, Cambridge, MA, 1992. 